\title{
RNA at the heart of gene expression: Special issue of BMB Reports 2017
}

Over the past several decades, our knowledge of RNAs has increased dramatically. In particular, the recent advances in next-generation sequencing, combined with crosslinking immunoprecipitation or other variants, has led to the expansion of a list of RNAs including long noncoding RNAs (IncRNAs) and small regulatory RNAs such as microRNAs, short interfering RNAs, and Piwi-interacting RNAs. Although only a small number of previously unappreciated RNA roles have begun to be revealed, it is a good time to introduce the recent RNA advances and their related functions to readers. This special issue on RNA invites three perspectives, five minireviews, and four research articles. The topics covered in this special issue include microRNA-mediated gene silencing, the molecular mechanism of nonsense-mediated mRNA decay (NMD), mRNA translation, mitochondrial noncoding RNA (ncRNA) transport, genome-wide analysis of ncRNAs, and alternative polyadenylation.

For the perspectives in this issue, three experts in Korea are invited: Prof. Chan-Seok Shin at Seoul National University, Prof. Seung-Jae V. Lee at Pohang University of Science and Technology, and Prof. TaeSoo Kim at Ewha Womans University. They summarize their recent works by highlighting their achievements in the field of RNA biology including a microRNA destabilization by noncanonical targets, the role of NMD in aging, and the dynamics of mRNAs and ncRNAs by H3K36 methylation, respectively.

This special issue also includes five invited mini-reviews. Dr. Myriam Gorospe and her colleagues at the National Institute on Aging summarize the up-to-date knowledge of mitochondrial transcripts and introduce recent progress in our understanding of how nuclear DNA-encoded RNAs are localized into mitochondria. In the sense that mitochondrial ncRNAs play an essential role in mitochondria function, it is very timely to give our readers a recent view on the importance and regulation of mitochondrial ncRNAs.

Prof. Fabrice Lejeune from the University Lille in France updates recent views on NMD, which is the best-characterized mRNA quality control pathway in eukaryotic cells. He introduces the multiple roles of NMD in a variety of biological functions, such as cellular apoptosis, autophagy, unfolded protein response, cell differentiation, organism development, and cellular defense. While studying NMD, it has been

https://doi.org/10.5483/BMBRep.2017.50.4.046

Received 27 February 2017 discovered that transcripts harboring nonsense mutation are degraded on mRNAs bound by a nuclear cap-binding complex (CBC). Because NMD is tightly coupled to a translation event, this observation indicates that $\mathrm{CBC}$ can direct mRNA translation. The details for $\mathrm{CBC}$-dependent translation and the differences in regulatory mechanisms between $\mathrm{CBC}$ and elF4E-dependent translations are reviewed by Dr. Incheol Ryu from Korea University in Korea, and myself. In addition, the efficiency of elF4E-dependent translation is dynamically controlled by 3'-untranslated region (3'UTR). Prof. Akio Yamashita from Yokohama City University School of Medicine and Prof. Osamu Takeuchi from Kyoto University in Japan introduce a variety of regulatory mechanisms for elF4E-dependent translation by 3'UTR-binding proteins.

Recent transcriptome-wide studies reveal that alternative polyadenylation (APA) functions as a previously unappreciated layer of a regulatory mechanism for gene expression. The mini-review by Prof. Jeongsik Yong and his colleagues at the University of Minnesota deals with the whole history of APA from the discovery to recent transcriptome-wide APA identification. They also introduce various methods, technologies, and bioinformatics algorithms, which have been adopted for APA isoform analysis.

Finally, four research groups in Korea contribute to the research articles in this special issue: Prof. Do Han Kim from the Gwangju Institute of Science and Technology, Prof. Eun Kyung Lee from The Catholic University of Korea, Prof. Hyeon Ho Kim from Samsung Advanced Institute for Health Sciences and Technology, Sungkyunkwan University, and Prof. Jin-Wu Nam from Hanyang University. The first three groups report the functional role of microRNA in myocardial hypertrophy, mitochondrial elongation, and proliferation and clonogenic ability, respectively. The last group analyzes IncRNAs expressed from zebrafish embryos and finds a tendency that IncRNAs harboring small open reading frames are significantly repressed by miRNAs as observed in mRNAs.

Although RNA is an emerging field, it is still in its infancy. It is my hope that this special BMB Reports issue on RNA will provide scientists entering this field with general concepts and inspiration about RNA.

Yoon Ki Kim ${ }^{1,2}$, Ph.D.

${ }^{1}$ Creative Research Initiatives Center for Molecular Biology of Translation, Korea University, ${ }^{2}$ School of Life Sciences, Korea University, Seoul 02841, Korea

E-mail: yk-kim@korea.ac.kr 